\title{
Using Computer Cognitive Atlas to Improve Students' Divergent Thinking Ability
}

\author{
$\mathrm{Na}$ Man, School of Information Engineering, Harbin University, China \\ Kechao Wang, School of Information Engineering, Harbin University, China \\ Lin Liu, School of Information Engineering, Harbin University, China
}

\begin{abstract}
Human society has entered the era of intelligence. Social development in the era of intelligence has spawned a large number of intelligent applications. Intelligent applications have put forward unprecedented requirements on the level of cognitive intelligence of machines, and the realization of machine cognitive intelligence depends on knowledge map technology. Divergent thinking is an important part of thinking and an important indicator for measuring innovative thinking. The research in this article found that after the experiment, the associated probabilities of the F values of fluency, flexibility, uniqueness, semantic divergence, graphical divergence, and problem divergence were $0.389,0.442,0.594,0.267,0.319$, and 0.478 , which were all greater than the significance level of 0.05 , that is, the divergent thinking ability of the experimental group has been significantly improved. The results of this study show that the use of computer cognitive maps can improve students' divergent thinking ability.
\end{abstract}

\section{KEYWORDS}

Artificial Intelligence, Cognitive Atlas, Divergent Thinking, Knowledge Atlas

\section{INTRODUCTION}

The development of artificial intelligence began in the late 1990s, and mainly focused on mining statistical models in statistics, which has also achieved today's machine learning. However, statistical learning alone is not enough to support intelligent implementation. Symbol knowledge is indispensable for intelligent implementation, because symbol knowledge enables the machine to have interpretability and also enables the machine to have the language "understanding" ability. Therefore, machines must learn to use symbol knowledge to solve problems and realize cognitive intelligence. Cognitive Atlas was born in this context. Cognitive Atlas is a research branch of computer science (Zhang, 2020; Di, 2016; Ma, 2018; Pardeller, 2017; Khatwani, 2018). It attempts to understand the essence of intelligence and realize a major technological breakthrough from cognitive intelligence system to cognitive intelligence system. At present, the application of cognitive maps is used in various aspects of life, bringing many conveniences to people. Creative thinking is a kind of creative thinking activity, that is, the thinking activity that opens new areas of human understanding and creates new achievements in human understanding. Creative thinking is based on the abilities of perception, memory, thinking, association, and understanding, and is a high-level mental activity characterized by comprehensiveness, exploratory and novelty, which requires people to put in hard mental work

DOI: 10.4018/JOEUC.20211101.oa25

This article published as an Open Access article distributed under the terms of the Creative Commons Attribution License (http://creativecommons.org/licenses/by/4.0/) which permits unrestricted use, distribution, and production in any medium, provided the author of the original work and original publication source are properly credited. 
(Fan, 2019; Ludolph, 2016; Zhang, 2017; Buckley, 2019; Mo, 2020). An achievement of creative thinking can only be obtained after long-term exploration, hard research, and even multiple setbacks. The ability of creative thinking can only be achieved after long-term accumulation of knowledge and quality. As for the process of creative thinking, it is not possible. Do not engage in a variety of thinking activities such as reasoning, imagination, association, and intuition (Qi, 2018).

Innovative thinking is the core quality of talents in the era of knowledge economy. Countries attach great importance to the cultivation of innovative thinking, and China also lists it as one of the core qualities of student development $(\mathrm{Li}, 2018)$. Divergent thinking is the core of innovative thinking. Cultivating divergent thinking is regarded as the goal of curriculum standards and the proper function of teaching materials. Divergent thinking is the core component of innovative thinking, and training divergent thinking is an important part of innovative talents. Cultivating divergent thinking will make students broaden their horizons, think quickly, be good at association, learn to observe, think, and answer problems from multiple angles and in all directions. Attaching importance to the cultivation of students' divergent thinking has become an important sign of educational modernization. Students' divergent thinking ability is of great significance to modern education (Omari, 2016; Bakhtavar, 2019; Beaty, 2018; Shahri, 2019; Xu, 2021; Raymond, 2017; Gong, 2016).

[1] Children's creative thinking is often assessed through a divergent thinking test. Aviva Berkovich-Ohana reviewed the literature on divergent thinking tests, arguing that the advantages of these tests include their solid theoretical basis, reliability, selective validity, and a large amount of literature that can be used to assist interpretation. The specific conclusions supported by Aviva Berkovich-Ohana's research are as follows. First, divergent thinking test scores can predict certain types of performance (such as writing), but not other areas (such as art). Second, the divergent thinking test has discriminative validity, but the traditional scoring methods (fluency, originality, flexibility) may be insufficient. When comparing the thinking test and the intelligence test, the ability level of the subject must be considered. Third, some personality characteristics (such as independence) and family variables (such as birth order, family size, age gap) are related to the performance of the Conceptual Creativity Test. Finally, the performance of divergent thinking can be influenced by models, including parental divergent thinking, motivation and reinforcement, task perception, environmental cues, stimulus characteristics, and age. Overall, research by Aviva Berkovich-Ohana shows that these tests help assess the potential of children's creative thinking (Liu, 2019; Gangurde, 2018). Empirical research on creativity focuses on the importance of divergent thinking, which helps to generate new solutions to loosely defined problems. Jing TENG used behavioral tasks and nearinfrared spectroscopy (NIRS) to detect the creativity and frontal cortex activity of a group of externally verified creative individuals (trained musicians) and demonstrated them in a graphically matched manner. Jing TENG's experiment 1 examined convergence and divergent thinking in intelligence and personality. Jing TENG's experiment 2 uses near-infrared spectroscopy to study the changes in forehead oxygenation and deoxyhemoglobin concentration during divergent thinking. The results of Jing TENG's experiment 1 show that musicians' creativity has improved, their language skills and their split personality have also improved, but after changing these two factors together, their divergent thinking has been enhanced. In Experiment 2, near-infrared spectroscopy showed that musicians had greater bilateral frontal lobe activity than non-musicians during divergent thinking (Jing, 2018; Luiza, 2018; Gacek, 2017; Ghanavati, 2017).

In the era of artificial intelligence, it is a very meaningful thing to use artificial intelligence to provide convenience to people. This paper brings up the study of using computer cognitive maps to cultivate students' divergent thinking ability. This article uses the literature review method to test cognitive Intelligence and knowledge maps are explained, and the method of controlled experiments is used to study the use of computer cognitive maps to cultivate students 'divergent thinking ability. The feasibility of this method is verified by studying and analyzing the comparison of students' divergent thinking ability before and after the experiment. Through the cultivation of this ability to improve the ability of students. 


\section{Proposed Method}

\section{Cognitive Atlas}

\section{Cognitive Intelligence and Cognitive Atlas}

The development of artificial intelligence has gone through three main stages so far: symbolic reasoning and perceptron, probability learning and knowledge base, deep learning and knowledge map. The current intelligent systems have reached or even surpassed the human level in terms of perception, but there are still many deficiencies in terms of interpretability, robustness, security and reliability. The development of cognitive AI systems is the future of AI development. This is not only the focus of deep learning in the future, but also the basis for the rise of the next generation of artificial intelligence.

Artificial intelligence has reached or surpassed human standards in the areas of perceptual intelligence such as "listening, speaking, and watching", but it is still in its infancy in the field of cognitive intelligence that requires external knowledge, logical reasoning, or domain migration. Cognitive Atlas will draw inspiration from cognitive psychology, brain science, and human social history, and combine technologies such as cross-domain knowledge atlas, causal reasoning, and continuous learning to establish an effective mechanism for stable acquisition and expression of knowledge, so that knowledge can be used by machines Understand and use, to achieve the key breakthrough from cognitive intelligence to cognitive intelligence. Cognitive Atlas aims to combine cognitive psychology, brain science, and human knowledge to develop a new generation of cognitive engines for knowledge atlas, cognitive reasoning, and logical expression to realize the evolution of artificial intelligence from cognitive intelligence to cognitive intelligence. Research on cognitive maps includes cognitive map representation, cognitive map construction, cognitive map reasoning, cognitive map applications, etc. Divergent thinking is not following the convention, seeking variation; using different methods or approaches to analyze and solve problems from different angles, in different directions, and to the given materials and information. The training of one problem with multiple solutions is a good way to cultivate students' divergent thinking. method. It can diverge vertically and horizontally, connect knowledge in series and communicate comprehensively, to draw inferences from one another.

Cognitive intelligence refers to the ability of machines to actively think and understand. They can realize self-learning, purposeful reasoning, and natural interaction with humans without human programming in advance. Human beings have language before concepts and reasoning, so concepts, consciousness, and ideas are all manifestations of human cognitive intelligence. Machines have a long way to go to achieve these capabilities.

\section{Knowledge Graph}

Knowledge graph is essentially a semantic web, which is a graph-like and related knowledge collection, which is often used to refer to a large-scale knowledge base. Because knowledge maps can intuitively and visually display information such as the subject's knowledge context and development history, early knowledge maps were mainly used in scientific research. In recent years, with the increasing degree of intelligence of information service applications, the scope of application of knowledge maps has become more extensive. At present, mature knowledge maps can be divided into three types: The first is the general domain knowledge graph. This kind of knowledge graph has no specific application areas, including rich information and a wide range of design areas. The second is the knowledge graph of a specific field. This kind of knowledge map is for a specific field and has a strong professionalism. The third is the cross-language knowledge graph. This knowledge graph describes knowledge in multiple languages. With the increasing number of English knowledge maps, the establishment of cross-language knowledge maps can give full play to the complementary role 


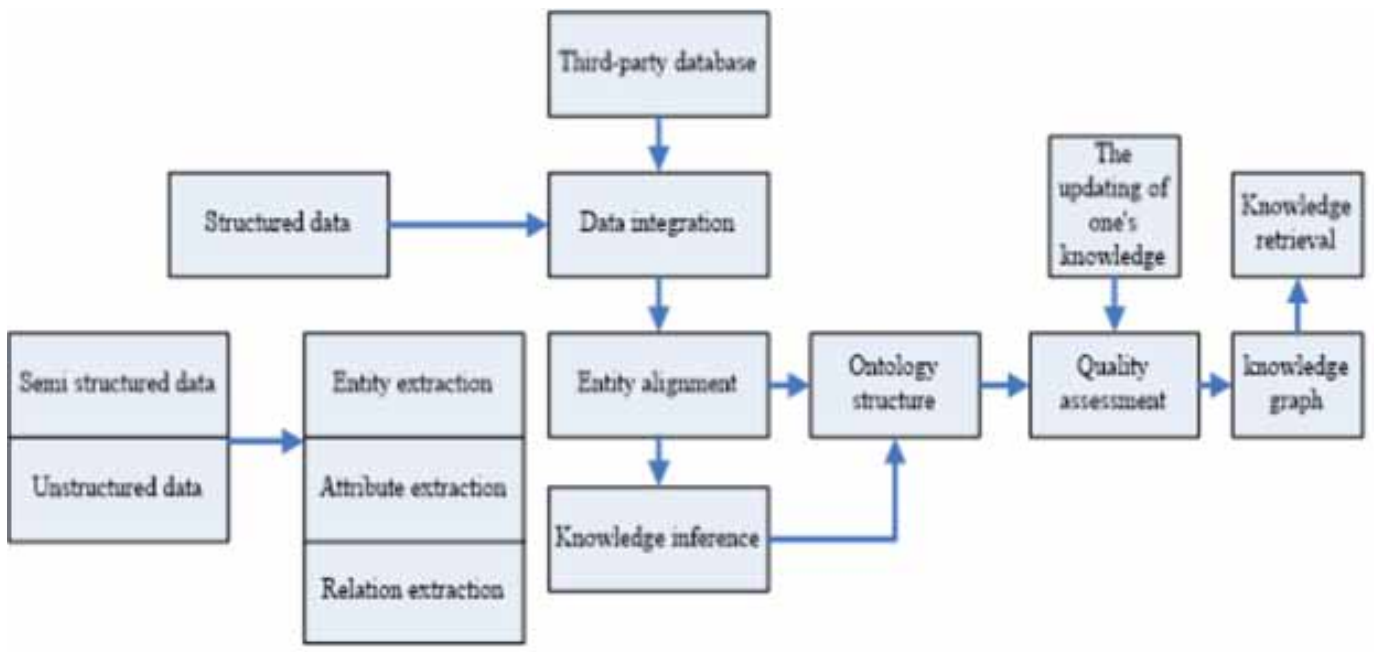

of knowledge expression in different languages, expand knowledge coverage and sharing, and make up for Lack of language knowledge.

\section{Knowledge Graph Construction Method}

Early knowledge map construction methods mainly used artificial methods to form general knowledge maps such as WordNe and ResearchCyc. In recent years, with the enrichment of network resources, knowledge map construction methods based on information extraction in the open domain have been widely adopted. The technical architecture of knowledge graph construction based on information extraction is shown in Figure 1.

From Figure 1, we can see that the technical architecture based on knowledge extraction of information extraction is based on the original data and according to the processing flow of knowledge resources, it can be divided into the following three stages: The first stage is knowledge extraction. Extract knowledge resources from existing structured and unstructured data; the second stage is knowledge fusion. Knowledge fusion is carried out through technologies such as data cleaning, entity disambiguation, coreference resolution, etc. to form a knowledge map. The third stage is knowledge evaluation, evaluating the quality of the existing knowledge map, and further digging hidden knowledge for knowledge reasoning.

\section{Knowledge Extraction}

Because knowledge is the basic element of the knowledge graph, knowledge extraction is the primary work to construct the knowledge graph. Knowledge includes three elements of entity (concept), attributes and relationships, and involves technologies such as entity extraction, relation extraction and attribute extraction. In the process of entity extraction, different structured data sources use different methods when extracting entities, entity attributes, and entity relationships. The following describes the information extraction technology of different structured data sources. Establish a knowledge graph meta-model with entity-relation-entity triples; use the knowledge graph model layer, knowledge resource ontology layer and knowledge resource instance layer as the hierarchical structure to establish a knowledge resource ontology model; combine the knowledge resource ontology entity with the knowledge. The resource ontology model constructs the knowledge resource ontology description model through the instance mapping relationship, and forms the corresponding knowledge graph storage. 


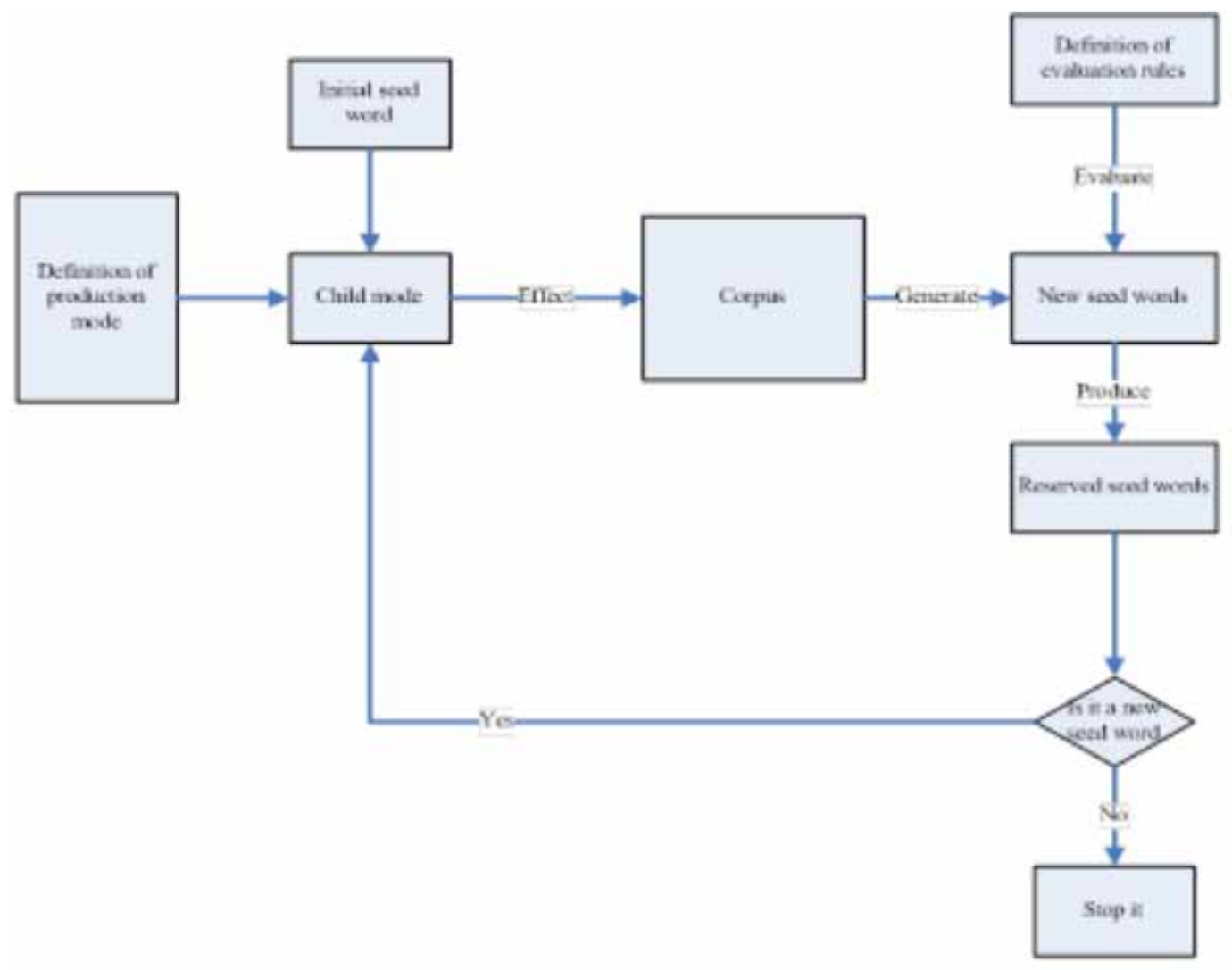

\section{Structured Data}

Extraction of unstructured data source entities can use machine learning methods, of which the commonly used method is bootstrapping method. The bootstrapping method only needs a seed with semantics to achieve entity mining. There is no necessary corpus training set in other machine learning methods. It is an unsupervised machine learning algorithm. The entity mining process it implements is shown in Figure 2.

It can be known from Figure 2 that the process of obtaining the named entity by the bootstrapping algorithm is as follows. The first step is to select the seed words for mining domain entities. The second step is to select the characters before and after the seed word as the attributes of the generated model, and select text between these characters. The generation model is based on the entity information and Corpus to determine text information. At the same time, you can set the nearby characters of the seed vocabulary as a window to get the text from the window. In the third step, after applying the seeded mode to the corpus, the extracted information will be determined according to the entity evaluation rules. Those entities that meet the judgment result and are needed will be retained; otherwise, they will be deleted. At the same time, these new entities will also be used as new seed words; finally, the new seed words are applied to the corpus. Repeat the previous steps until no new seed words are generated. The core of bootstrapping algorithm is the selection of seed words. The selection of seed words determines the generation of new seeds. The characteristics of machine learning methods are mainly high efficiency and high accuracy. At the same time, the generation mode and evaluation rules 
in the algorithm are also very important. They are usually defined based on the entity information of the corpus.

\section{Semi-Structured Data}

Semi-structured data sources are mainly different encyclopedia data. The concept of entities is usually described in the form of web pages. The web pages contain information modules of entities. Web crawlers are usually used to extract entities and entity attributes. Most of the entity data in the encyclopedia is displayed in the form of "attribute.value". When extracting real attributes from the entity data page, you only need to write adapter parsing to the information module of the page to complete it. For the entity attributes extracted from different encyclopedia data sources, the attribute values need to be merged because of the similarity between the entities.

\section{Structured Data}

Structured data includes existing domain ontology, XML files, tabular data, and relational database resources. For these structured resources, the corresponding data needs to be parsed for different ways of storing its data. Two types of data, OWL and XML, are usually obtained by toolkits: use the dom $4 \mathrm{j}$ toolkit to extract the category and category relationship of the XML data format; use the Jena toolkit to perform the categories and categories on the OWL and RDF format data Relation extraction. Aiming at the relational database resources, it can be directly obtained or data analysis methods can be used to more accurately obtain the relationship between entities after excluding invalid information. Commonly used data analysis methods are the four-table chi-square test, which is based on the ternary representation of the ontology structure (concept.relation.concept), and analyzes two of the conceptual entities to remove the uncertain factors in the database. In summary, in the information extraction step, different entities, entity attributes and entity relationship extraction algorithms need to be adopted according to the structured degree of the data source: structured non-data, such as nonrelational databases, whose data resource system is clear and It is rich in content and usually needs to be obtained by different toolkits or software according to the way its structured data is stored; semi-structured data usually refers to different encyclopedia data sources, the concept of which exists in webpages, and webpage information is also There are certain rules. The concept of search usually describes the concept, concept attributes and attribute values in the form of information modules. The web crawler can directly obtain this information to complete the information supplement and expansion of the concept attributes; non-structural In generalized data, entity extraction and entity relationship extraction are usually performed in different ways.Entity extraction usually uses machine learning methods, including unsupervised, semi-supervised and supervised algorithms. Relation extraction is usually based on rules and statistics. Combined with rules-based and statistical methods, commonly used relationship extraction algorithms include SVM algorithms and CRF algorithms.

\section{Knowledge Fusion}

Knowledge fusion is an important step in the construction and representation of knowledge spectrum. Through knowledge extraction, original knowledge resources can be obtained. Because the source of knowledge is extensive and the quality is difficult to determine, it may contain a lot of ambiguity, ambiguity, redundancy and even wrong information, so the original knowledge must be cleaned and fused. Knowledge fusion is a higher degree of knowledge organization. The technologies involved are entity disambiguation, coreference resolution, and multiple data source mergers. Entity disambiguation refers to the technique of eliminating different meanings of the same entity. In a real language environment, a concept often refers to multiple objects. Common methods of entity disambiguation include bag-of-words model, semantic model, social network model, and encyclopedic knowledge model. Coreference resolution is a technique that eliminates the same meaning of multiple entities. 


\section{Knowledge Evaluation}

The evaluation of knowledge quality is an important factor related to the quality of the knowledge map. The significance is to quantify the credibility of knowledge in order to eliminate low-value knowledge, retain high-reliability knowledge, and improve the quality of the knowledge map. Credibility refers to the credibility of data and contains five quality dimensions: data source, data publisher reputation, data credibility, verifiability, and data use license. At present, knowledge evaluation faces the following problems: First, due to the complexity of network information and the limitations of technical conditions, the original knowledge resources extracted on the open domain will inevitably contain redundancy or errors. Reasoning, the other knowledge obtained is also without quality assurance. Therefore, quality evaluation is required for the information such as entities and associations in the knowledge map. Finally, as the number of knowledge graphs increases, the conflicts between them also increase. How to resolve conflicts and get rough and fine is an important work to build a global knowledge map.

\section{Divergent Thinking}

Divergent thinking is a higher-level form of human thinking. Divergence is explained in modern Chinese dictionaries as being centered on one point and spreading around. Therefore, divergent thinking is also called diffusion thinking, different thinking or radiant thinking. Divergent thinking can be understood as starting from known conditions or facts, getting rid of the limitations of original knowledge, seeking answers from different paths and directions, reorganizing existing information, and generating information that matches the problem to solve the problem. It can be understood as: generating new information from the information given. Divergent thinking can break through the mindset and inspire people to observe and solve problems more thoroughly from a possible perspective. It is a process in which people's thinking activities unfold through multiple directions, levels, and perspectives. At present in China, there are two representative views on the understanding of divergent thinking: one is that divergent thinking is a form of thinking. When classifying the thinking form, it is divided into two categories: divergent thinking and concentrated thinking according to the direction of thinking. Another view is to consider divergent thinking as the quality of thinking, and think that the flexibility of thinking is actually divergent thinking. This article considers divergent thinking as a form of thinking.

\section{Divergent Thinking and Concentrated Thinking}

Divergent thinking and concentrated thinking are two forms of thinking to solve problems. Concentrated thinking can also be referred to as convergent thinking and convergent thinking, which refers to a way of thinking that solves problems raised and masters knowledge by analyzing various information and according to existing experiences and methods. Its characteristic is that the thinking is very concentrated, and given information is thinking in one direction to generate new information. Divergent thinking and concentrated thinking are essentially a pair of opposing and unified thinking. The thinking process of concentrated thinking will use more analysis, synthesis, generalization and other methods, while the thinking process of divergent thinking will use more comparison, analogy, deduction and other methods. As far as solving a biological problem, students need to think about the relevant knowledge points through the information given by the topic, and this process is divergence. Students may produce different problem solving methods, which is also divergent. However, to determine the best answer for students, they need to use concentration and analyze the divergent ideas through comprehensive analysis and other methods to obtain the answer. The two are infiltrated and dialectically unified. Therefore, it is not that concentrated thinking is not important, but that at present, people place too much emphasis on concentrated thinking and ignore the role of divergent thinking. 


\section{Divergent Thinking and Innovative Thinking}

Divergent thinking is often associated with innovative thinking. Some people even equate the two, but in fact they are different. Innovative thinking can also be called creative thinking, which refers to being able to creatively clarify the nature of the object to be researched and produce unique thinking results on this basis. The organic combination of divergent thinking and concentrated thinking is creative thinking. In fact, the basic form of innovative thinking is divergent thinking, which is the most critical thinking requirement of human beings when innovating. If there is no divergence of thinking when innovating, it means that the original mindset is difficult to break through, and of course, there is no need to talk about innovation. Then the training of the model is realized through the method of machine learning. The main function of divergent thinking is to make people dare to find differences, and when thinking about problems, they can conceive from multiple sides and constantly seek new things. Without divergent thinking, it will not provide a lot of new clues for solving problems, and it will also reduce the possibility of innovation. In fact, the perfect combination of concentrated thinking and divergent thinking can better exert creativity.

\section{Characteristics of Divergent Thinking}

\section{Fluency}

Fluency measures the speed at which a person's mind diverges. It can be regarded as an indicator of the "quantity" of divergent thinking and the basis of divergent thinking. The better fluency means that people can express more concepts in unit time, which is a reflection of fluent thinking and agility. To cultivate students' fluency of divergent thinking, we must pay attention to guide students to accumulate knowledge and collect information, especially in the era of globalization and informationization.

\section{Flexibility}

The flexibility of divergent thinking is an indicator of the "qualitative" nature of divergent thinking. Flexibility is actually the key to divergent thinking. The so-called flexibility refers to the flexible use of knowledge and multi-angle thinking on issues, including borrowing, crossover, replacement, and integration of related concepts and content. We must deeply understand the meaning of the word "flexibility", that is, in addition to paying attention to the "what" characteristics of things, we must also consider its "what" purpose when thinking about problems. When training the flexibility of divergent thinking, we must pay attention to the breadth, it requires rich imagination and association.

\section{Uniqueness}

Uniqueness is the essence of divergent thinking, and also the purpose of divergent thinking, manifested as a novel component of thinking. Uniqueness is also called novelty or originality, which is the sign and basic characteristic of divergent thinking. In the process of divergent thinking, negation of authority or inclination often occurs, but this negation is not blind, nor is it specifically for negation. The purpose of negation is to create new ones. Of course, uniqueness is relative. If a person solves a problem, whether the solution is unique or not. The standard is not whether the method proposed by the individual has been proposed, but whether the solution to the problem is unique to the person. In other words, uniqueness is aimed at solving two aspects of the problem, one is the subject and the other is the object. If it is novel to the subject, it is mainly to promote the development of the individual. If it is novel to the object, it is mainly to promote the development of society.

\section{Experiments}

\section{Research Methods}

This research involves the following main research methods: 
(1) Literature method: Research on the basic theory of divergent thinking, and provide a theoretical basis for the research on the rational application of divergent thinking to the teaching of college mathematics. At the same time, theoretical research is made on the measurement of students' learning interest, classroom effects, and measurement of divergent thinking, and questionnaires and measurement scales are made to accurately evaluate and measure before and after experiments.

(2) Questionnaire survey method: First, a questionnaire survey is conducted on the development of students' divergent thinking ability in college mathematics teaching. Then, in this study, a questionnaire was compiled based on previous studies through literature analysis. Use the "Learning Interest and Attitude Scale" in Educational Measurement and Evaluation to investigate changes in student learning interest. Use "Creativity Test" in Psychological Experiment Guide to measure the three dimensions of divergent thinking ability.

Based on previous experience, this article compiles a questionnaire for divergent thinking of students. Then test and analyze to find out the discrimination, reliability and validity of the questionnaire to form the final questionnaire. Before the beginning of teaching practice, a questionnaire survey was conducted on selected experimental and control classes to collect data and analyze the status quo, and at the same time provide support for the development of subsequent teaching practices. After the end of the teaching practice, a questionnaire was written again to test the two classes, and the pre-test and post-test data were analyzed.

(3) Teaching experimental research: The practical use of divergent thinking in experimental classes in teaching. The experimental design and the control group were compared before and after the experimental design.

\section{Research Object}

Collecting and accumulating purely scientific and technological communication media materials also has far-reaching influence and important significance on the development of science and technology and economic construction. By collecting data and accumulating knowledge, wrong conclusions or wrong guesses made in the past can also be corrected. Collecting information and accumulating knowledge is the prerequisite for intelligence research and the basis for scientific and technological decision-making. Collect information and accumulate knowledge, ask questions $₫$ collect information ( ) make predictions. Discuss the importance of collecting data from the perspective of information theory: only when there is input, there is output. In this paper, all the students from class 1 and 2 of a computer major in a university are selected as research objects, 2 classes are experimental classes, and 1 class is a control class. Among them, the number of students in the control group was 40 and the number of students in the experimental group was 41 . Through the comparison before and after the experiment, this paper studies and analyzes the training results of divergent thinking ability of students using computer cognitive maps. Intelligent semantic filtering and review of text content, built-in the most complete vocabulary, intelligent identification of multiple variants: deformation, phonetic change, traditional and simplified variants, and precise semantic disambiguation.

\section{Experimental Design}

Experimental process During the implementation of the experiment, the experimental class uses the computer's cognitive map to develop the ability of infiltrating divergent thinking in the teaching of advanced mathematics. The method of cultivating the divergent thinking described above is used to teach the students in the experimental class. The development of thinking and dynamic research on the development of students' divergent thinking. The teachers of the control class still follow the traditional teaching, and do not pay special attention to the cultivation of divergent thinking. After the experimental period, the data were analyzed and compared. 
Table 1. Reliability analysis of three factors

\begin{tabular}{|l|l|l|c|}
\hline Reliability statistics & Semantic divergence & Graph divergence & $\begin{array}{c}\text { Problem } \\
\text { divergence }\end{array}$ \\
\hline Cronbach's Alpha value & 0.840 & 0.896 & 0.817 \\
\hline Several items & 4 & 3 & 4 \\
\hline
\end{tabular}

\section{Discussion}

\section{Analysis of the Status Quo of Students' Divergent Thinking}

\section{Reliability Analysis of the Questionnaire}

To further understand the reliability and validity of the questionnaire, a reliability test is required. The reliability test method is usually performed by Cronbach'SAlpha coefficient. In this paper, the reliability coefficient value greater than 0.7 is considered to be credible. Three factors were selected for reliability analysis. The results are shown in Table 1.

As can be seen from Table 1, the Cronbach'SAlpha coefficients of semantic divergence, graphical divergence, and problem divergence are $0.840,0.896$, and 0.817 , all of which are greater than 0.7 , and have a high degree of credibility, indicating that the topic is valid and credible.

\section{Analysis of the Status Quo of Divergent Thinking}

Because the number of items contained in different dimensions is different, the mean of the three dimensions and three indicators is calculated through data processing. At the same time, the three indexes and three dimensions of divergent thinking were analyzed by SPSS. The results are shown in Table 2 and FIGS. 3 and 4.

Combining Table 2 and Figure 3 and Figure 4 shows that from the three indicators, the average values of fluency, flexibility, and uniqueness are 69.63, 29.28, and 4.24, but from the average of each question, they are 6.329 and 2.653 And 0.410. From the three dimensions, the mean values of semantic divergence, graph divergence and question divergence are 56.54, 18.11, 28.66, and the average value of each question is $14.135,6.037$, and 7.165. From the index correlation of divergent thinking, all three indexes have a positive correlation, and the correlation between flexibility and uniqueness is the highest, the correlation between fluency and flexibility is second, and the correlation between fluency and uniqueness at last. Words and thoughts are completely asymmetrical; words and thoughts are completely inconsistent. People always think that they can gain insights into others and the world through learning, observation, accumulation, and analysis. But when they look back, they find that it is themselves who cannot be analyzed. It is precisely their own differences in thinking that have misled

Table 2. Statistics analysis of students' divergent thinking description

\begin{tabular}{|l|l|l|l|l|}
\hline & N & Mean value & Item & $\begin{array}{l}\text { Mean value of each } \\
\text { question }\end{array}$ \\
\hline Fluency & 81 & 69.62 & 11 & 6.329 \\
\hline Flexibility & 81 & 29.18 & 11 & 2.653 \\
\hline Uniqueness & 81 & 4.51 & 11 & 0.410 \\
\hline Semantic divergence & 81 & 56.54 & 4 & 14.135 \\
\hline Graph divergence & 81 & 18.11 & 3 & 6.037 \\
\hline Problem divergence & 81 & 28.66 & 4 & 7.165 \\
\hline
\end{tabular}




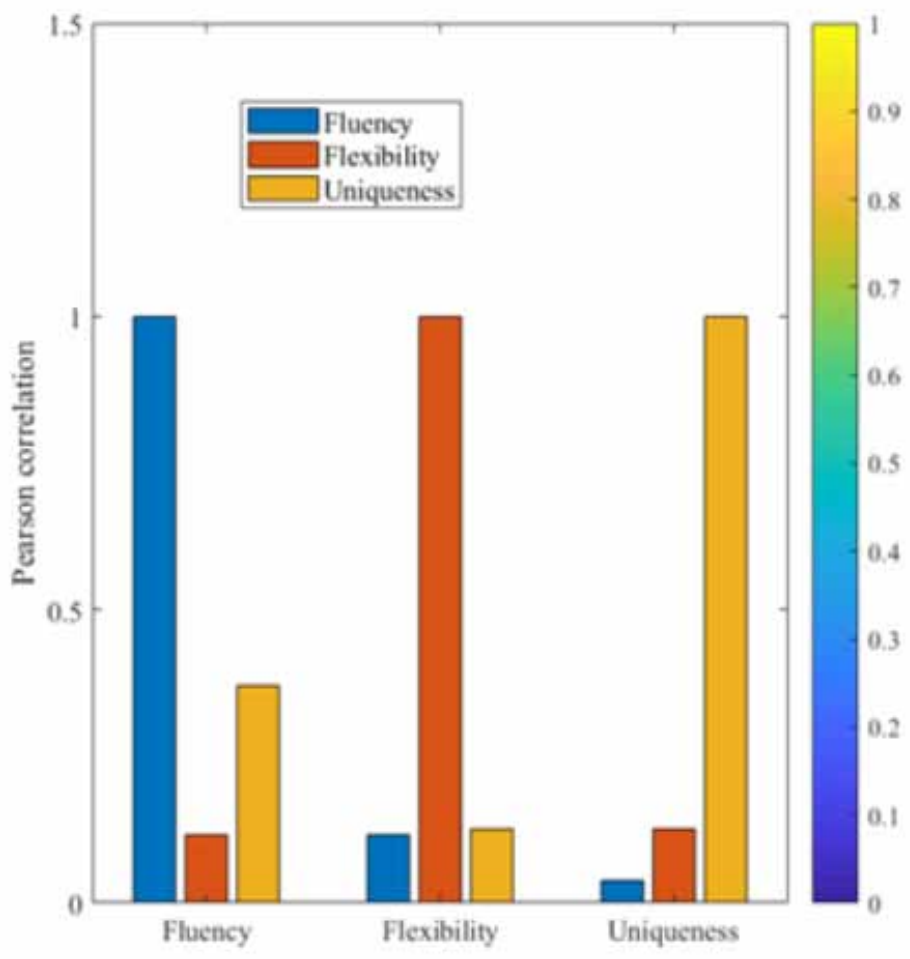

the entire careful and rigorous consideration. From the perspective of divergent thinking, there is a significant positive correlation between the three dimensions. Graphical divergence has the highest correlation with problem divergence, followed by semantic divergence and problem divergence, and semantic divergence and graph correlation are last. But as a whole, there is a positive correlation between both indicators and dimensions.

\section{Analysis of Cultivation Results of Using Computer Cognitive Atlas to Improve Students' Divergent Thinking Ability}

\section{Results and Analysis of the Questionnaire Before the Experiment}

Before the experiment, an independent sample T test was performed on the results of the students' questionnaires to see if there were significant differences between the two classes. The test results are shown in Table 3 and Figure 5.

As can be seen from Table 3 and Figure 5, the mean values of fluency are 68.65 and 70.58, the mean values of flexibility are 28.75 and 29.60 , the mean values of uniqueness are 4.27 and 4.75 , the mean values of semantic divergence are 57.20 and 55.87, and the graph diverges. The mean values are 17.17 and 19.04, respectively, and the mean values of the problem divergence are 27.30 and 30.02 , respectively. The students of classes 15 and 16 have little difference in the mean of each dimension and index. In addition, it can be seen from the table that the associated probabilities of the F values of fluency, flexibility, uniqueness, semantic divergence, graphical divergence, and problem divergence are $0.676,0.333,0.388,0.770,0.231$, and 0.429 , which are all greater than significant The sex level 
Figure 4. Correlation analysis of divergent thinking dimensions

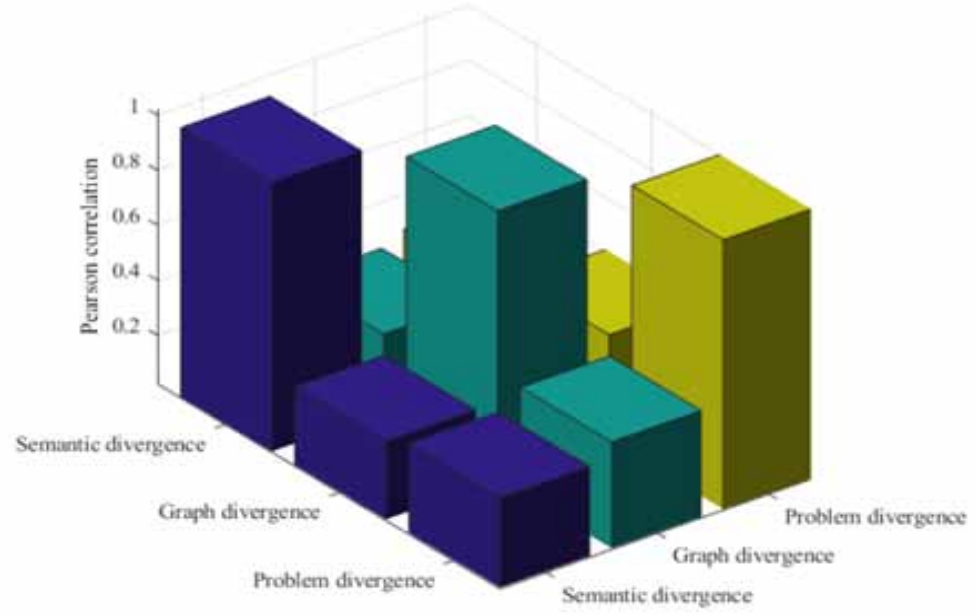

Table 3. Results and analysis of questionnaire before the experiment

\begin{tabular}{|c|l|l|l|l|l|l|l|}
\hline $\begin{array}{l}\text { Mean value of } \\
\text { control group }\end{array}$ & $\begin{array}{l}\text { Standard } \\
\text { deviation } \\
\text { of control } \\
\text { group }\end{array}$ & $\begin{array}{l}\text { Standard } \\
\text { deviation of } \\
\text { experimental } \\
\text { group }\end{array}$ & $\begin{array}{l}\text { Mean } \\
\text { value of } \\
\text { experimental } \\
\text { group }\end{array}$ & $\begin{array}{l}\text { Standard } \\
\text { deviation of } \\
\text { experimental } \\
\text { group }\end{array}$ & $\begin{array}{l}\text { Mean } \\
\text { standard } \\
\text { error of } \\
\text { experimental } \\
\text { group }\end{array}$ & $\begin{array}{l}\text { Concomitant } \\
\text { probability of } \\
\text { F value }\end{array}$ & $\begin{array}{l}\text { Concomitant } \\
\text { probability of } \\
\text { T-statistics }\end{array}$ \\
\hline 68.65 & 13.09 & 2.07 & 70.58 & 15.01 & 2.345 & 0.676 & 0.539 \\
\hline 28.75 & 4.98 & 0.831 & 29.6 & 3.84 & 0.788 & 0.333 & 0.387 \\
\hline 4.27 & 1.51 & 0.24 & 4.75 & 1.77 & 0.276 & 0.388 & 0.194 \\
\hline 57.2 & 9.83 & 1.555 & 55.87 & 9.6 & 1.5 & 0.77 & 0.542 \\
\hline 17.17 & 4.2 & 0.664 & 19.04 & 4.88 & 0.762 & 0.231 & 0.068 \\
\hline 27.3 & 7.64 & 1.209 & 30.04 & 6.48 & 1.013 & 0.429 & 0.087 \\
\hline
\end{tabular}

is 0.05 , so the zero hypothesis of the $\mathrm{T}$ test cannot be rejected, indicating that there is no significant difference between the students in the two classes in these three dimensions and above the three indicators. The $\mathrm{T}$ test results when the variances are equal show that the probability associated with the T statistic of fluency, flexibility, uniqueness, semantic divergence, graphical divergence, and problem divergence are $0.539,0.387,0.194,0.542,0.068,0.087$, significance, respectively. The average level 


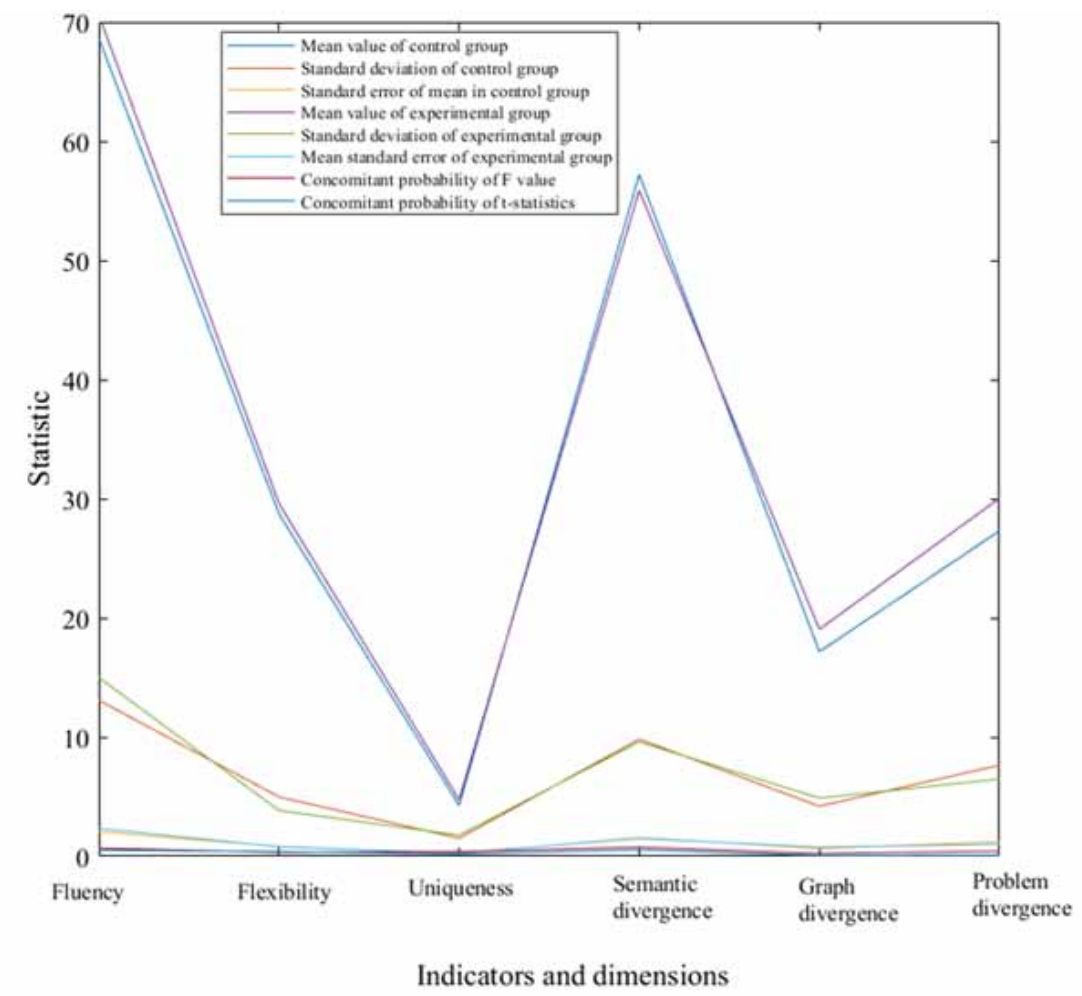

is greater than 0.05 . Therefore, the null hypothesis of the $\mathrm{T}$ test cannot be rejected, indicating that the students in the two classes have no significant differences in these three indicators and dimensions.

\section{Results and Analysis of the Questionnaire After the Experiment}

After the experiment, an independent sample $\mathrm{T}$ test was performed on the students' questionnaire results to see if there was a significant difference between the two classes. The test results are shown in Table 4 and Figure 6.

Combining Table 4 and Figure 6 shows that after the experiment, the mean values of fluency are 65.06 and 72.89 , the mean values of flexibility are 30.35 and 33.16 , the mean values of uniqueness are 4.46 and 4.96 , and the mean values of semantic divergence are 56.58 and 60.00 . The mean values of the divergences of the graphs are 17.02 and 20.08, and the mean values of the problem divergences are 26.27 and 30.93. The students of classes 15 and 16 look at the mean of each dimension and index. The difference is larger than the previous test, and there are differences. In addition, it can be seen from the table that the associated probabilities of the F values of fluency, flexibility, uniqueness, semantic divergence, graphical divergence, and problem divergence are 0.389, 0.442, 0.594, 0.267, 0.319 , and 0.478 , which are all greater than significance. At the level of 0.05 , the zero hypothesis of the $\mathrm{T}$ test cannot be rejected, indicating that there is no significant difference between the students in the two classes in these three dimensions and above the three indicators. The results of the $\mathrm{T}$ test when the variances are equal show that the probabilities associated with the T statistics of fluency, flexibility, semantic divergence, graphic divergence, and problem divergence are $0.001,0.016,0.027$, 0.001 , and 0.001 , respectively, and the significance level is less than 0.05 . Therefore, rejecting the null hypothesis of the $\mathrm{T}$ test, it means that the students of the two classes have significant differences 
Table 4. Questionnaire results and analysis after the experiment

\begin{tabular}{|c|l|l|l|l|l|l|c|}
\hline $\begin{array}{l}\text { Mean value of } \\
\text { control group }\end{array}$ & $\begin{array}{l}\text { Standard } \\
\text { deviation } \\
\text { of control } \\
\text { group }\end{array}$ & $\begin{array}{l}\text { Standard } \\
\text { deviation of } \\
\text { experimental } \\
\text { group }\end{array}$ & $\begin{array}{l}\text { Mean } \\
\text { value of } \\
\text { experimental } \\
\text { group }\end{array}$ & $\begin{array}{l}\text { Standard } \\
\text { deviation of } \\
\text { experimental } \\
\text { group }\end{array}$ & $\begin{array}{l}\text { Mean } \\
\text { standard } \\
\text { error of } \\
\text { experimental } \\
\text { group }\end{array}$ & $\begin{array}{l}\text { Concomitant } \\
\text { probability of } \\
\text { F value }\end{array}$ & $\begin{array}{l}\text { Concomitant } \\
\text { probability of } \\
\text { T-statistics }\end{array}$ \\
\hline 65.06 & 10.945 & 1.579 & 72.89 & 12.423 & 1.774 & 0.389 & 0.001 \\
\hline 30.35 & 5.930 & 0.856 & 33.16 & 5.305 & 0.758 & 00.442 & 0.016 \\
\hline 4.46 & 1.320 & 0.191 & 4.96 & 1.443 & 0.206 & 0.594 & 0.027 \\
\hline 56.58 & 6.619 & 0.955 & 60.00 & 8.236 & 11.180 & 0.267 & 0.001 \\
\hline 17.02 & 3.970 & 0.0573 & 20.08 & 4.494 & 0.642 & 0.319 & 0.001 \\
\hline 26.27 & 7.324 & 1.057 & 30.93 & 6.097 & 0.871 & 0.478 & 0.001 \\
\hline
\end{tabular}

Figure 6. Questionnaire results and analysis after the experiment

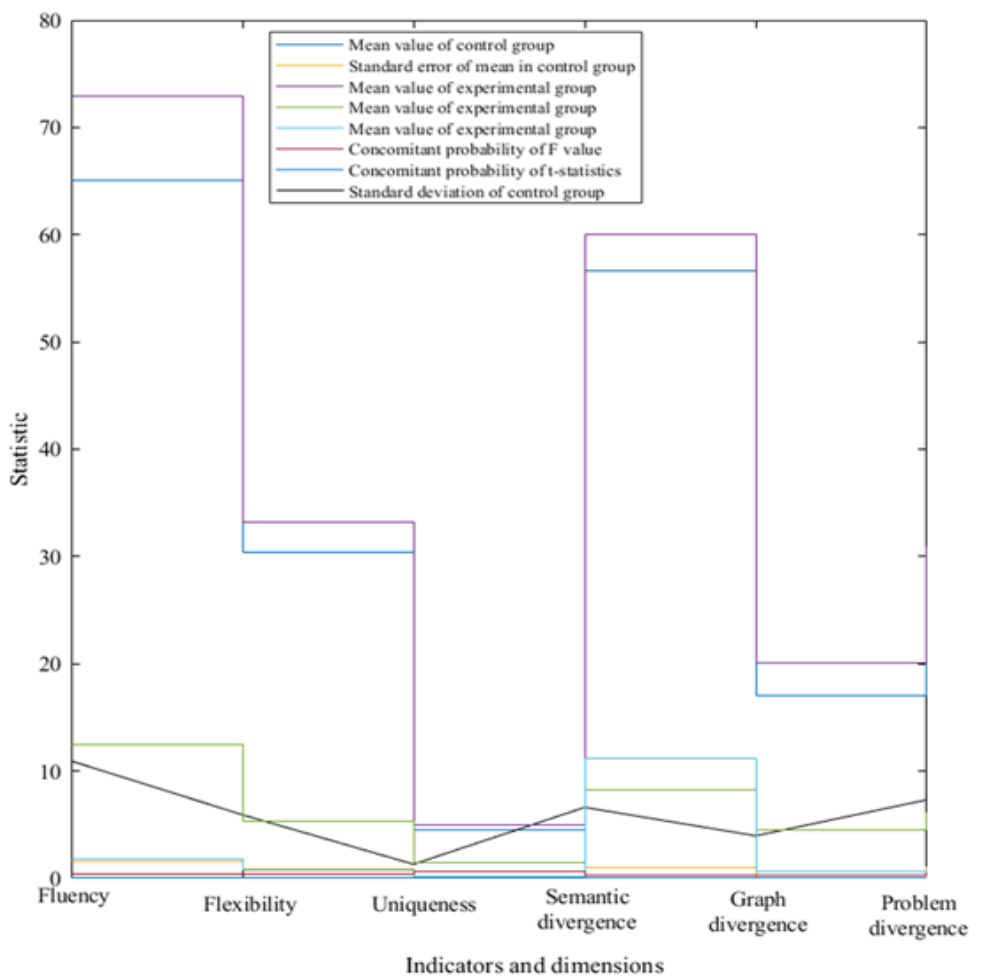

in fluency, flexibility, semantic divergence, graphical divergence, and problem divergence. The probability associated with the unique T statistic is 0.078 , which is greater than the significance level 
of 0.05 . The zero hypothesis of the $\mathrm{T}$ test cannot be rejected, indicating that there is no significant difference in the uniqueness of the students in the two classes.

\section{Conclusion}

With the development of artificial intelligence, the development of cognitive intelligence has also shown an ever-changing momentum, and computer cognitive maps have begun to be applied to all aspects of daily life. We know that divergent thinking ability is an important part of innovation ability, and innovation ability is very important for this era, and improving the cultivation of students' divergent thinking ability has also become a research hotspot. This article uses computer knowledge map to divergent thinking ability of students The culture was carried out, and the feasibility of the method was verified by experiments.

This article analyzes the current situation of students' divergent thinking. It can be seen that among the three indicators, fluency scores highest, flexibility is second, and uniqueness is last. Of the three dimensions, the score of the semantic divergence is the highest, the problem divergence is the second, and the graphic divergence is the last. And among these three indicators, the fluency score is significantly higher than the other two indicators. In the three dimensions, the score of semantic divergence is also significantly higher than the other two dimensions, which indicates that the development of divergent thinking of students is uneven.

After the experiment, this study found that there was no significant difference in the uniqueness of thinking between the control class and the experimental class, and the results of the divergent thinking test showed that the experimental group was compared with the control group on the indicator of uniqueness of thinking. It has not been significantly improved, but from the overall perspective of the experiments in this article, it is still possible to find that the divergent thinking ability of the experimental group has improved significantly. From this, we can know that the method of cultivating students' divergent thinking using computer cognitive map is feasible of.

\section{ACKNOWLEDGMENT}

This work was supported by Natural Science Foundation of Heilongjiang Province (Grant No. LH2019F046), Harbin science and technology innovation talents research project (Grant No. 2016RAQXJ013) 


\section{REFERENCES}

Buckley, P., Noonan, S., Geary, C., Mackessy, T., \& Nagle, E. (2019). An empirical study of gamification frameworks. Journal of Organizational and End User Computing, 31(1), 22-38. doi:10.4018/JOEUC.2019010102

Di, L., Zhang, H., Kang, C., \& Guo, T. (2016). Erps evidence for the relationship between fluid intelligence and cognitive control. Neuroreport, 27(6), 379-383. doi:10.1097/WNR.0000000000000547 PMID:26934284

Fan, X., Wang, F., Wang, F., Gong, W., \& Liu, J. (2019). When rfid meets deep learning: Exploring cognitive intelligence for activity identification. IEEE Wireless Communications, 26(3), 19-25. doi:10.1109/ MWC.2019.1800405

Gangurde, S. R., \& Patil, S. S. . (2018). Benchmark product features using kano - qfd approach: a case study. Benchmarking An International Journal, 25(11). 10.1108/BIJ-08-2016-0131

Jing, T., Shen, W., \& Hao, N. (2018). The role of cognitive control in divergent thinking. Xinli Kexue Jinzhan, 26(3), 411. doi:10.3724/SP.J.1042.2018.00411

Khatwani, G., \& Srivastava, P. R. (2018). Impact of information technology on information search channel selection for consumers. Journal of Organizational and End User Computing, 30(3), 63-80. doi:10.4018/ JOEUC.2018070104

Li, A. (2018). A practical approach to constructing a knowledge graph for cybersecurity. Engineering, 4(1), 53-60. doi:10.1016/j.eng.2018.01.004

Liu, W., Ma, H., \& Walsh, A. (2019). Advance in photonic crystal solar cells. Renewable \& Sustainable Energy Reviews, 116, 109436. doi:10.1016/j.rser.2019.109436

Ludolph, R., Allam, A., \& Schulz, P. J. (2016). Manipulating google's knowledge graph box to counter biased information processing during an online search on vaccination: Application of a technological debiasing strategy. Journal of Medical Internet Research, 18(6), e137. doi:10.2196/jmir.5430 PMID:27255736

Luiza \& Fabisiak. (2018). Web service usability analysis based on user preferences. Journal of Organizational \& End User Computing.

Ma, H., Zhang, X., Ju, F., \& Tsai, S. B. (2018). A study on curing kinetics of nano-phase modified epoxy resin. Scientific Reports, 8(1), 3045. doi:10.1038/s41598-018-21208-0 PMID:29445228

Pardeller, S., Frajo-Apor, B., Kemmler, G., \& Hofer, A. (2017). Emotional Intelligence and cognitive abilitiesassociations and sex differences. Psychology Health and Medicine, 22(8), 1001-1010. doi:10.1080/13548506 .2016.1255766 PMID:27852112

Qi, S., Wu, Y., Peng, L., Dong, L., \& Hui, S. (2018). Mining summaries for knowledge graph search. IEEE Transactions on Knowledge and Data Engineering, PP, 30(99), 1-1. doi:10.1109/TKDE.2018.2807442

Zhang, X., Liu, X., Li, X., \& Pan, D. (2017). Mmkg: An approach to generate metallic materials knowledge graph based on dbpedia and Wikipedia. Computer Physics Communications, 211, 98-112. doi:10.1016/j. cpc.2016.07.005

Zhang, X., Zang, C., Ma, H., \& Wang, Z. (2020). Study on removing calcium carbonate plug from near wellbore by high-power ultrasonic treatment. Ultrasonics Sonochemistry, 62, 104515. Advance online publication. doi:10.1016/j.ultsonch.2019.03.006 PMID:31753547 